\title{
Granular flow down a flexible inclined plane
}

\author{
Prasad Sonar ${ }^{1, \star}$, Ishan Sharma ${ }^{1, \star \star}$, and Jayant Singh ${ }^{2, \star \star \star}$ \\ ${ }^{1}$ Department of Mechanical Engineering. \\ ${ }^{2}$ Department of Chemical Engineering. \\ Mechanics and Applied Mathematics Group, Indian Institute of Technology Kanpur, Kanpur, UP 208016, India.
}

\begin{abstract}
Discrete and continuous systems are commonly studied individually, but seldom together. Indeed, granular flows are typically studied through flows over a rigid base. Here, we investigate the behaviour of granular flows over an inclined, flexible base. The flexible base is modeled as a rigid platform mounted on springs and has one degree of freedom. The base vibrations are introduced by the flow. We simulate such flows through a discrete element method and compare with experiments. We find that a flexible base increased the upper limit of the inclination up to which a steady flow is possible by at least 3 degrees. This stabilized zone may have important implications in applications such as conveyor belts and chutes.
\end{abstract}

\section{Introduction}

In most literature on granular flows over bumpy or smooth bases, the base is considered rigid and fixed for convenience (e.g[1-4]). The rigid base may be actively moved or vibrated to introduce energy into the system. However, in real applications like chute flow, and transport and conveying systems, the base's motion is driven by the granular flow. A vibrating base may couple with the flow in a manner which increase collisional energy dissipation. This can affect the flow considerably.

Here, as a first step, we introduce flexibility in the base by supporting it upon springs. We investigate the flow over such a base for various spring stiffnesses through discrete element (DE) simulations and experiments. We define a stabilized regime in parameter space for which flexibility of the base increases the dissipation of kinetic energy by collisions among the grains and, therefore, slows down the material making the flow steady, whereas it would be accelerated for rigid bases. At lower inclinations, we find that the flexible base may be employed to control the manner in which the granular flow is initiated. These features may have important applications in the handling and transport of grains. Finally, we attempt to characterize the onset of unsteady granular flow in our study by making contact with stability analysis performed for viscous fluid flow over soft solids [8] and for dense granular flow over rigid inclined plane $[3,4]$.

\section{Methodology}

Figure 1 shows our experimental setup. It consists of two $40 \mathrm{~mm}$ wide acrylic channels. We use steel balls with

\footnotetext{
^e-mail: prasads@iitk.ac.in

$\star \star$ e-mail: ishans@iitk.ac.in

$\star \star \star$ e-mail: jayantks@iitk.ac.in
}

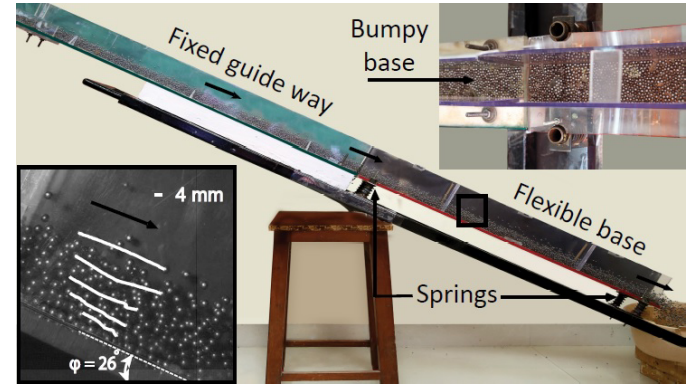

Figure 1. The experimental setup consists of a fixed guideway and the spring supported flexible base.

$4 \mathrm{~mm}$ diameter as flowing granular material. The same balls are employed to make the base bumpy. First, the $2 \mathrm{~m}$ long guideway is fixed to the iron frame, and it guides the flowing particles into the second $1 \mathrm{~m}$ long 'active'channel whose base is made flexible by supporting it with springs on either ends. During experiments, the active channel oscillates up and down in its first mode of vibration primarily. When the flow has matured, the pitching motion of the base, corresponding to second mode of vibration, is observed to be small and, hence, ignored in this study.

During flow, we maintain a flow thickness of $40 \mathrm{~mm}$, which corresponds to approximately 10 steel ball diameters, by controlling the outlet knob of the hopper. We perform experiments on angles of inclination $\varphi$ varying between $22^{\circ}$ and $30^{\circ}$. We also vary the stiffnesses of the supporting springs. This compressibility $\zeta$ is the inverse of the spring's stiffness $k_{s}$ scaled by the stiffness $k_{n}$ of the steel balls. We employ springs with stiffness values varying from 2 to $15 \mathrm{~N} / \mathrm{mm}$ that corresponds to $\zeta$ varying from 70 to 260 . The contact stiffness of steel balls $k_{n}=400 \mathrm{kN} / \mathrm{m}$ as found by [6]. We employ particle tracking and image processing techniques to obtain quantitative data from the flow. 
We also simulate a three-dimensional granular flow with periodic boundary conditions (PBC) along the direction of the flow, as shown in Fig. 2. We employ DE simulations, whose details are available in Bhateja et al. [2]. The flow consist of spherical, cohesion-less, monodispersed, inelastic (coefficient of restitution $e=0.88$ ), soft (non-dimensional normal spring stiffness $k_{n}=2 \times 10^{5}$ ) and rough (friction coefficient $\mu=0.5$ ) grains. The base is made rough and bumpy, and is supported on a linear spring in a direction normal to the flow. The spring stiffness $k_{s}$ is kept lower than that of the grain's. The compressibility $\zeta$ in simulations, equivalent to experiments, is the ratio of the grain stiffness $k_{n}$ to the spring stiffness $k_{s}$. We have not introduced any damper with the supporting springs. Thus, energy only dissipates through collisions among particles and with the base. Our simulations follow the same flow initiation process as in Silbert et al. [1].
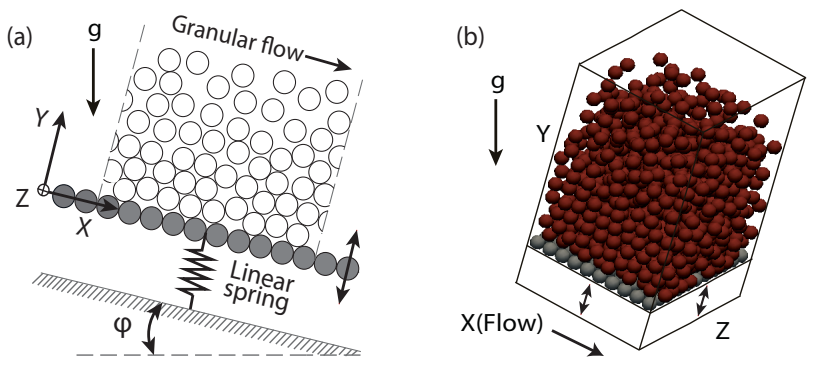

Figure 2. Granular flow over flexible base with one degree of freedom in direction normal to the inclined channel. Periodic boundary condition are imposed along the inclined plane (xdirection). (a) A two-dimensional schematic representation, and (b) a three-dimensional DE simulations of the flow.

In our simulations we have employed depth distribution of momentum as a criterion to differentiate between conditions of flow and no-flow. Figure 3(a) shows the distribution of momentum through the depth of the granular flow at various base inclinations. At lower inclinations, momentum is carried only by the top few layers, and the remaining grains are nearly static. However, at higher inclinations the momentum is well distributed through the flow depth. We define flow to have initiated when the minimum momentum per unit depth over half the depth at a cross-section is at least $10 \%$ of the total momentum at that cross-section. A flow is said to be steady when the total kinetic energy $\left(\mathrm{E}_{K}^{T}\right)$ along the inclined plane is the same for all time. This happens when the power input by gravitational force is balanced by that lost to collisional dissipation and base vibration.

\section{Results: Simulations}

A typical result as shown in Fig. 3(b). We observe that on a rigid base flow accelerates when $\varphi$ exceeds $29^{\circ}$, but on a flexible base with $\zeta=80$, the flow remain steady even at $\varphi=32^{\circ}$. In passing we note that our result for steady granular flow on a rigid base with flow depth equaling 10 grain diameters are in agreement with Silbert et al. [1].

Our simulation results are summarized in Fig. 4 in terms of the parameters $\varphi$ and $\zeta$. The state of the flow is
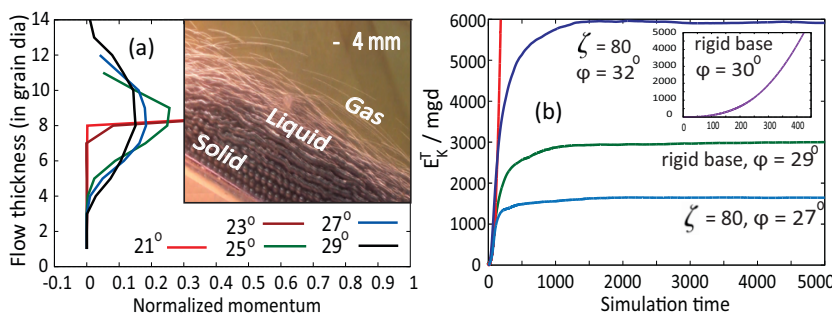

Figure 3. Flow criterion. (a) Momentum distribution through the depth at different angle of inclination $\varphi$. Inset shows states of flowing material, and is indicative of momenta carried by grains. (b) Kinetic energy of the granular flow on rigid and flexible bases. Inset shows increase in $\mathrm{E}_{K}^{T}$ on rigid base at $\varphi=30^{\circ}$. Here, energy is normalized using grain's mass $m$, diameter $d$ and the constant of gravitational acceleration $g$.

represented in terms of the angle of inclination $\varphi$ and compressibility $\zeta$. The inclination angle $\varphi$ is varied between $21^{\circ}$ to $32^{\circ}$ and $\zeta$ ranges between 0 and 100 , with 0 corresponding to a rigid base, and 100 representing the softest base. The stabilized regime is the zone in parameter space

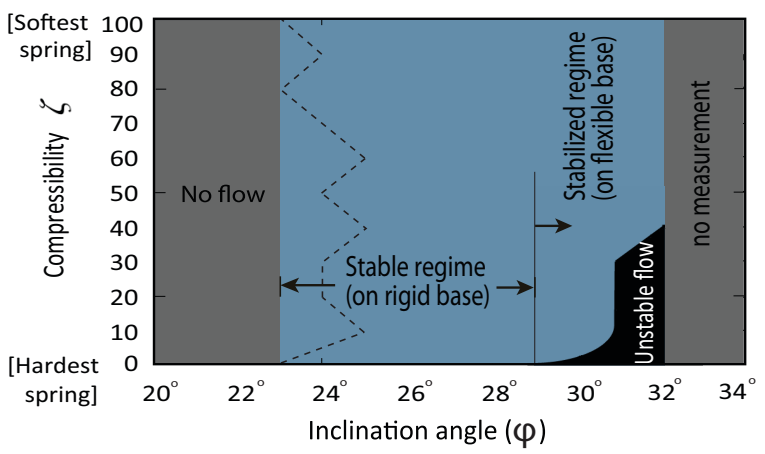

Figure 4. Behavior of granular flow down a channel with inclination $\varphi$ supported by springs with compressibility $\zeta$. Dashed line represents the inclination angle at which the flow initiates.

where the flow remains steady on the flexible bases. It is computationally expensive to track very rapid flows and, hence, inclinations above $32^{\circ}$ are not simulated. It is very likely that some of these rapid flows, perhaps at greater $\zeta$, are steady. Thus, the right boundary of the stabilized regime should not be understood as vertical.

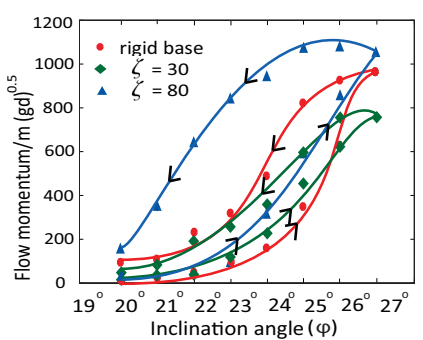

Figure 5. Effect of spring compressibility $\zeta$ on flow initiation and cessation. $\mathrm{Y}$ axis is scaled using grain's mass $m$, diameter $d$ and the constant of gravitational acceleration $g$. Markers indicate simulation points.

As shown in Fig. 5, flow starts and stops at different $\varphi$ when the inclination is increased and then decreased at the same constant rate. This happens due to the inertia of the grains. As the granular material is known to be hysteritic in nature, such behavior is not surprising. The width of hysteritic loop is affected by the base conditions which, as Fig. 4 suggests, may be used to reduce hysteresis. This will allow better control over the flow. 
Figure 6 shows various flow properties at $\varphi=26^{\circ}$ for different compressibilities $\zeta$. We find from Fig. 6(a) that on a rigid base, grains at the bottom do not contribute to the momentum of the flow. However, on flexible bases, these grains get energized due to base vibrations. Hence, even if the variation of number density with depth is comparable for various $\zeta$, as shown in Fig. 6(b), grains at the bottom are more agitated when the base is flexible, indicating more collisions. The velocity profile in Fig. 6(c) further confirms that grains near the bottom are in motion where the base is flexible.
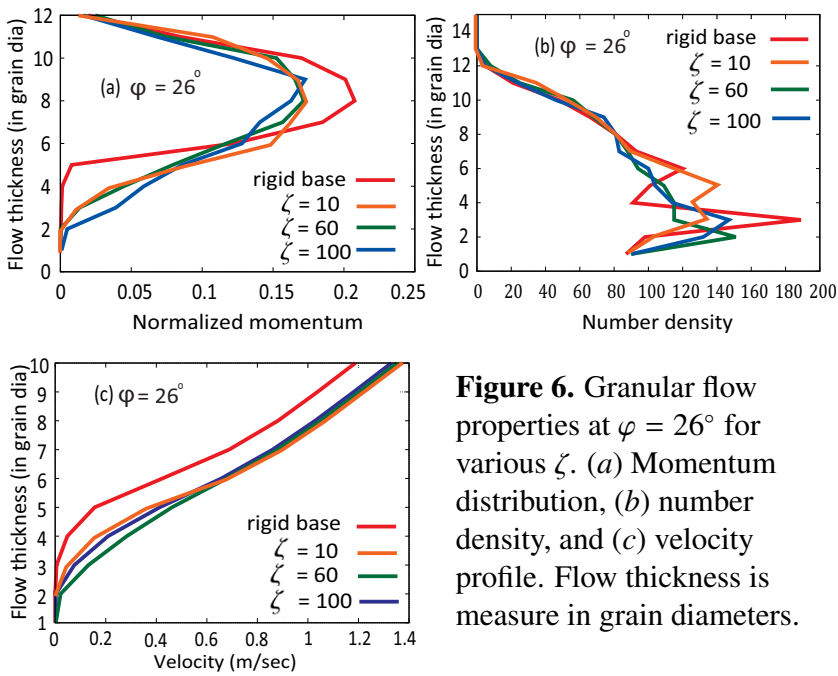

Figure 6. Granular flow properties at $\varphi=26^{\circ}$ for various $\zeta$. (a) Momentum distribution, $(b)$ number density, and $(c)$ velocity profile. Flow thickness is measure in grain diameters.

To understand the source of the stabilized regime in Fig. 4, we investigate the effect of base flexibility on the granular flow. Consider first the simple one-dimensional computational experiment shown in Fig. 7. A grain is dropped on a stack of other grains resting on a spring mounted base. As shown, the impacting grain bounces many more times on a rigid base than on a flexible base. This suggests that energy is lost faster on a flexible base in contrast to rigid base. Together with increase in numbers of collisions (see Sect. 5) for flows over flexible bases this explains stabilization of rapid flows at high inclination by flexible bases.

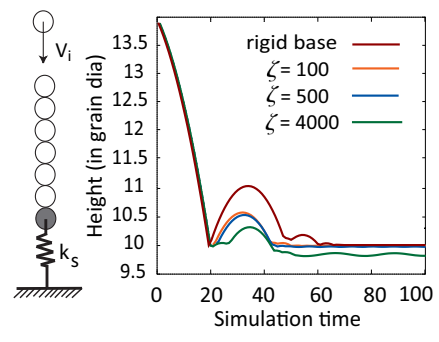

Figure 7. A grain bouncing on a stack of grains resting on a spring. Various compressibilities are considered.

\section{Results: Experiments}

Experimental and computational velocity profiles for various inclinations on rigid base are compared in Fig. 8. We find a qualitative agreement. Figure 9 shows experimentally measured total kinetic energy $\mathrm{E}_{K}^{T}$ at five different location along the inclined plane. Rigid and flexible bases inclined at various $\varphi$ are considered. We have tracked $\mathrm{E}_{K}^{T}$ at five different locations along the flow channel. Flow enters the $1 \mathrm{~m}$ long active base after traveling trough the $2 \mathrm{~m}$

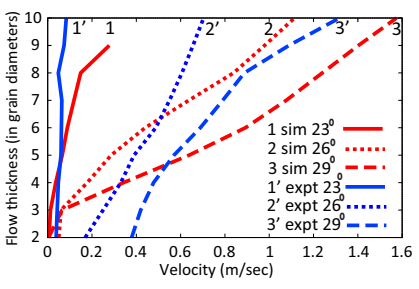

Figure 8. Comparison between experiments and simulations of velocity profiles of flows on a rigid base.

long guideway. We observed steady state regime between inclinations $\varphi=25^{\circ}$ and $28^{\circ}$ on rigid base. At higher inclinations $\left(\varphi=29^{\circ}\right), \mathrm{E}_{K}^{T}$ of the flow increases on a rigid base, but remains constant for flows over flexible base. This difference is in line with the predictions of simulations about the stabilizing effect of a flexible base on a granular flow.

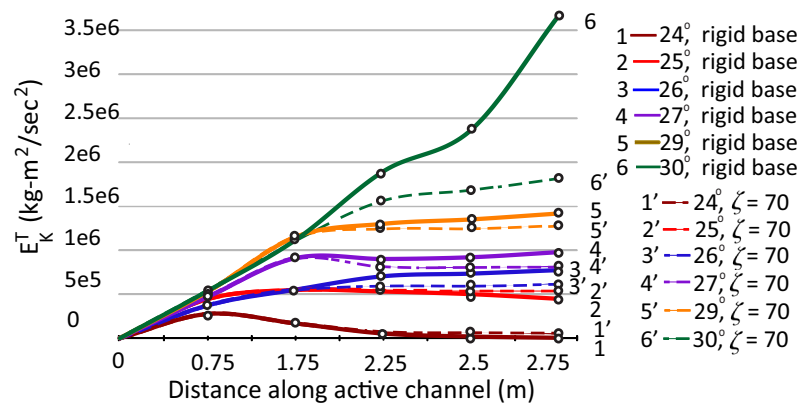

Figure 9. Kinetic energy of the flow $\mathrm{E}_{K}^{T}$ at various locations along the length for flows over rigid and flexible bases at various inclinations $\varphi$.

Figure 10 shows the variation of layer-wise kinetic energy $\mathrm{E}_{K}^{L}$ through the depth for flow over a flexible base inclined at $\varphi=29^{\circ}$ when compressibility $\zeta=70$. It is seen that top grains accelerate while those at the bottom decelerate. Predictably, grains at the top appear to be unaffected by base conditions. However, grains at the bottom reflect the effect of a flexible base in terms of a reduced kinetic energy.

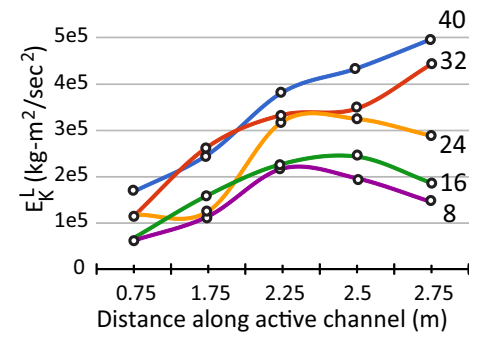

Figure 10. Depth-wise distribution of $\mathrm{E}_{K}^{L}$. Labels 8 - 40 indicate depth (in mm) at which measurement is taken.

\section{Discussion}

We now investigate energy dissipation in such flows through simulations by comparing $\mathrm{E}_{K}^{T}$ of the flow at $\varphi=$ $29^{\circ}$ on a rigid and flexible bases. We have seen that the kinetic energy, $\mathrm{E}_{K}^{T}$ of the flow is lower on a flexible base. We next trace the reason for the difference $\Delta \mathrm{E}_{K}^{T}$ between the total kinetic energy of flows over rigid and flexible bases.

We find that the total number of grain contacts $\left(\mathrm{N}_{C}^{T}\right)$ per unit time step are higher in flows on a flexible base. The energy stored in the spring is very small compared to $\mathrm{E}_{K}^{T}$ and, so, may be ignored. Hence, increased $\mathrm{N}_{C}^{T}$ is the reason for the greater dissipation in flows over flexible bases. Independent of base conditions, approximately $85 \%$ of contacts occur among particles $\left(\mathrm{N}_{C}^{P}\right)$ and $15 \%$ are 
due to collisions with the base $\left(\mathrm{N}_{C}^{B}\right)$. As shown in Fig. 11, comparing $\mathrm{N}_{C}^{T}$ for flows at $\varphi=29^{\circ}$ on a rigid base with that over a flexible bases, we find that $\mathrm{N}_{C}^{P}$ increases from 330 to 414 and $\mathrm{N}_{C}^{B}$ from 52 to 74 per time step, i.e., increment of $25 \%$ and $42 \%$, respectively. At $\varphi=26^{\circ}$, as the flow is denser, $\mathrm{N}_{C}^{P}$ increases by $50 \%$, but $\mathrm{N}_{C}^{B}$ remains about the same. It is important to note that collisions at $\varphi=29^{\circ}$ are much more dissipative than those of flows at $26^{\circ}$, due to higher grain velocities. The energy stored in the spring does not vary much with inclination and changes only by $10 \%$ for compressibilities $(\zeta)$ varying between 10 and 100 . Thus, we believe that base flexibility increases the number of inter-grain collisions which, in turn, cause more dissipation in the flow, thereby stabilizing it.

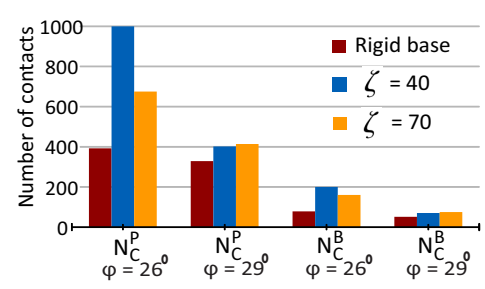

Figure 11. Comparison of number of inter-grain collisions $\left(\mathrm{N}_{C}^{P}\right)$ and number of collisions with base $\left(\mathrm{N}_{C}^{B}\right)$. See the text for details.

While we have observed increased energy dissipation in flows over flexible bases in both experimentally and in simulations, a steady flow in experiments is seen for narrow region. We believe that this happens because (a) channel used in experiments is not long enough, and (b) limitations on various grain and spring parameters available for experiments. Steel balls used in experiments are much harder compared to the grains simulated. We are forced to use grains with higher density, like steel balls, to allow us to use a variety of soft springs. Lighter grains like glass beads or plastic balls would force us to use softer springs that are hard to find and tend to fail easily in shear. At the same time, simulations with high stiffness coefficients $k_{n}$ are computationally prohibitive.

\section{Stability analysis}

Here we make an initial attempt to connect onset of unsteady flow in our system with the stability of Newtonian fluids down deformable inclined surfaces [8]. The first correction to the wave-speed $c_{1}$, was found by [8] to be

$$
c_{1}=i[((6 / 5) \operatorname{Re}-\cot \varphi)-9 \Gamma H],
$$

where $R e$ is the Reynolds number, and $\Gamma$ is the solid elasticity parameter, which is a ratio of the viscous stress in flowing material to the elastic stress in the supporting flexible structure. The flow is unstable if the imaginary part of wave-speed, $c_{1}$ is positive. On a rigid base, where $\Gamma=0$, the free surface undergoes long wave instability when $(6 / 5) \operatorname{Re}>\cot \varphi$. On a deformable solid, the free surface instability is completely stabilized if $(9 \Gamma H)>$ $((6 / 5) R e-\cot \varphi)$. This defines the stability criterion. In our simulations, $\Gamma=\mu(d u / d y)(1 / E)$, with $E=\left(k_{n} H / v^{2} l^{2}\right)$, being the equivalent modulus for our spring supported base. Parameters $\mu, v, H$ and $l$ are, respectively, bulk viscosity, volume fraction, equivalent thickness of elastic solid, and the length of the base. Here, $d u / d y$ and $v$ are found from simulations and $l$ is taken to be 10 grain diameters, and $\mu$ is obtained from kinetic theory [7] (eq. 7.161). The thickness $H$ drops out in calculation and so need not be estimated.

The stability conditions of [8], when applied to our granular flow, predict that flow on a rigid base becomes unstable for $\varphi \geq 26^{\circ}$. On flexible base with $\zeta=40$ and 70 , flow is predicted to be stable. Thus, the theoretical predictions of [8] also note an increase in stability in inclined flows in the presence of a deformable base. However, a quantitative match is not seen, as is to be expected given the difference in flowing material and base motion.

The spatial stability of steady uniform flow over a rigid inclined base was investigated by [3, 4]. Employing depth averaging and $\mu(I)$-rheology predicted that steady-uniform flows become unstable when $F>F_{c}=2 / 3$. Where $F$ is Froude number $\left(F_{r}=\underline{\mathrm{v}} /(g H \cos \varphi)^{0.5}\right)$ and $F_{c}$ is critical Froude number. Parameters $g, \mathrm{v}$ and $H$ are constant of gravitational acceleration, depth-average flow velocity and flow thickness respectively. In our simulations, flow on rigid base becomes unstable for $\varphi \geq 26^{\circ}$, and remain stable for $\varphi \leq 28^{\circ}$ on flexible base with $\zeta=40$. The extension of these studies to granular flow over deformable surfaces remains a goal for further study.

\section{Conclusion}

We have shown that a flexible base stabilizes granular flow, which otherwise would accelerate on a rigid base. We found that a flexible base energizes the lower grains in the flow which results in more grain collisions. Increased collisions among the particles and with the base dissipate kinetic energy, stabilizing flows at higher inclination angles. Finally, we made a first step towards relating the stabilization of granular flows over flexible bases to stability of Newtonian fluids flowing over soft solids. A proper understanding of how base flexibility affects granular flows will allow us to better control them in engineering applications.

\section{References}

[1] Silbert L. E., Ertas D., Grest G. S., Halsey T. C., Levine D., and Plimpton S. J., Phys. Rev. E. 65, 051307 (2002).

[2] Bhateja A., Sharma I., and Singh J. K., Phys. Fluids. 28, 043301 (2016).

[3] Forterre Y. and Pouliquen O., J. Fluid Mech. 486, 2150 (2003).

[4] Gray J. M. N. T. and Edwards A. N., J. Fluid Mech. 755, 503-534 (2014).

[5] Cundall P. A. and Strack O. D. L., Geotechnique 29, No. 1, 47-65 (1979).

[6] Mishra B. K. and Rajamani R. K., Appl. Math. Modelling. 16, 598-604. (1992).

[7] Rao K. K. and Nott P. R., An Introduction to Granular Flow (Cambridge University Press, UK, 2008) 490.

[8] Shankar V. and Sahu A. K., Phys. Rev. E. 73(1), 016301 (2006). 